\title{
VALIDACION DEL PROGRAMA ORQUIDEAS Y GIRASOLES UNA PROPUESTA DE TRABAJO EN SALUD MENTAL INFANTIL
}

\author{
María Elsa Bustamante Soto
}

\begin{abstract}
RESUMEN
Este artículo muestra los resultados obtenidos a partir del estudio que realizó REDINFA * para validar una propuesta de trabajo en salud mental infantil.

El programa "Orquídeas y Girasoles» es un programa que responde a dos objetivos básicos que es el de apoyar a niños afectados por la violencia política y favorecer su desarrollo psicosocial. La presente validación se realizó con niños de 7 a 14 años de edad.

El programa trabaja bajo tres ejes fundamentales, que son la persona, la familia y la comunidad. En cuanto a la persona se busca que los niños reconozcan sus habilidades y destrezas y a su vez sus defectos y virtudes para así superar situaciones difíciles y mirar con optimismo el futuro. En cuanto a familia, es la de que reconozcan su historia familiar y poder comprender el porque el alejamiento de su comunidad de origen y finalmente en cuanto a comunidad, es el de revalorar sus costumbres y hábitos de vida de su anterior lugar de residencia, y que en el actual espacio se puedan dar cuenta que son capaces de influir en su desarrollo.

El programa se inicia con un primer momento de tomar contacto con la población objetivo y a través de actividades lúdicas para desarrollar los nexos de confianza y así iniciar el programa que constó de 25 sesiones en las que se trabajo tres ejes: Que valioso soy. Así es mi familia y Viviendo en comunidad.

La validación se lleva a cabo mediante la aplicación de una ficha de indicadores que tiene 6 áreas, que son: Relaciones interpersonales expresión de pensamientos y sentimientos, valoración personal, identidad. Visión de futuro y relación con su comunidad. De estas se encontró que la de Identidad tuvo mejores resultados no siendo así con las de valoración personal y la de relaciones interpersonales.
\end{abstract}

Palabras Claves: Salud mental, violencia política familia, comunidad, niño

\section{ABSTRACT}

This article shows the results obtained starring from the study that REDINFA * carried out to validate a work proposal in infantile mental health.

The program "Orchids and Sunflowers» it is a program that he/she responds to two basic objectives that it is the one of to support children affected by the political violence and to favor their development psychosocial. The present validation was carried out with children from 7 to 14 years of age.

The program works under three fundamental axes that are the person, the family and the community. As for the person it is looked for that the children recognize Their abilities and dexterities and in turn Their defects and virtues stop this way to overcome difficult situations and lo look with optimism the future. As for family, it is the that they recognize their family history and to be able to understand the because of the estrangement of their origin community and finally as for community, it is the one of revaluing their customs and habits of life of their previous residence place, and that in the current space They can be given bill that they are able to influence in their development.

The program begins with a first moment to lake contact with the population objective and through Playful activities to develop nexuses of trust and this way to begin the program that consisted or 25 sessions in those that you work three axes: That valuable I am. it is This way my family and living in community.

The validation is carried out by means of the application or a record or indicators that you/they have 6 areas that are: Relate Interpersonal, expression or thoughts and feelings. personal valuation. identify. Of these it was found that of identity had better results: not being this way with those of personal valuation and that of interpersonal relationships.

Key words: mental health, political violence, family, community, boy.

* REDINFA, Red para la Infancia y la Familia 


\section{POR QUÉ UNA PROPUESTA DE ATENCION EN SALUD MENTAL INFANTIL}

El año 1992, REDINFA Red para la Infancia y la Familia - Perú preocupada por la situación de violencia política por la que atravesábamos los peruanos y preocupada especialmente por cómo esta violencia estaba afectando a los niños, inicia la ejecución de su Programa de Atención en Salud Mental Infantil PASMI con el objetivo de apoyar en la recuperación emocional de los niños directamente afectados por la violencia política.

Con el transcurrir de las acciones y en el proceso de formación de Equipos Locales en las zonas de intervención del proyecto, fuimos perfilando la propuesta de atención a niños, la cual debía ser una herramienta que pudiera facilitar la recuperación de la Salud Mental de los niños y al mismo tiempo favorecer su desarrollo psicosocial, pues sabíamos que la violencia política había afectado a los niños a tres niveles: El afectivo, el cognitivo y el relaciona!'

La violencia política y el desplazamiento habían dejado en los niños sentimientos de miedo e inseguridad, nostalgia, depresión, en muchos casos irritabilidad, ansiedad y duelos no resueltos, así como sentimientos de culpa por pensar que no habían podido hacer nada frente a lo que vivieron. Con el transcurrir del tiempo estos sentimientos se han ido reflejando en el tipo de relación interpersonal que establece marcada básicamente por la desconfianza y temor, el aislamiento y las reacciones agresivas: pero también en sus perspectivas de vida, determinando una pobre o nula visión de futuro y problemas de atención y concentración que repercuten fuertemente en su desempeño escolar limitando sus posibilidades de incorporal mayores herramientas que les permita enfrentar su situación.

Pero los efectos psicosociales de la violencia política también se dieron en el entorno familiar y social de los niños, ámbitos de los que no podemos desvincularlos, por lo que en consecuencia no podemos decir que los efectos evidenciados en los sujetos están disociados de aquellos que se observan en la sociedad en su conjunto. Las secuelas dejadas en los individuos y vividas de manera peculiar en función a las circunstancias en las que se dieron, tiene sus raíces en la sociedad, la que al mismo tiempo se ve alterada por la influencia que ejercen los individuos en su dinámica con el medio. Por tanto, los efectos que podemos encontrar a nivel individual se verán de alguna manera reflejados en la sociedad y viceversa.

\section{LA PROPUESTA DE TRABAJO CON NIÑOS}

Las constataciones y reflexiones antes expuestas nos llevaron a pensar que debíamos trabajar un programa que contemplara los tres niveles (tres ejes): Persona, Familia y Comunidad; de tal manera que en el primer nivel se pudiera con-seguir que los niños se reconozcan como seres con cualidades y defectos. pero también con habilidades y destrezas (recursos) que les permiten superar las situaciones difíciles, identificando los sentimientos que les generaron y les generan las situaciones por las que han pasado y finalmente poder reincorporar su capacidad de mirar al futuro con optimismo.

El segundo eje (familia) se elaboró con la intención de que a través de las actividades los niños pudieren conocer y valorar su historia familiar, comprender por qué su familia tuvo que salir de la comunidad de origen y por qué en su casa no se habla de la época en que sucedió todo esto. Podrán también expresar los sentimientos que les genera lo que sucede al interior de su familia y comprender que para los adultos también es doloroso y muy duro recordar esas experiencias.

Los objetivos del tercer eje estaban dirigidos a tratar de ver la comunidad de origen como era realmente, revalorando su cultura y costumbres. Al mismo tiempo se buscaría que los niños puedan ver que en el nuevo barrio hay también cosas buenas y malas, y que al 
formar parte de él tienen la capacidad de influir en su desarrollo.

El programa consta de 2 sesiones introductorias en las que se conversa con los niños sobre sus expectativas y lo que se va a hacer durante el tiempo que dure el taller y se establece con ellos las normas de convivencia para el grupo. Luego se sugiere una primera etapa en la que el promotor o promotora pueda realizar actividades lúdicas y técnicas de arte que permitan generar confianza en el grupo y la habituación al trabajo colectivo. Por último se plantean 25 sesiones distribuidas en tres ejes: Que valioso soy, Así es mi familia y Viviendo en comunidad.

\section{ANTECEDENTES DE LA VALIDACION}

El año 1994 el PASMI inició una experiencia de trabajo con niños en el Asentamiento Humano Cangalla. Esta experiencia tenía por objetivo que a partir del trabajo directo pudiéramos implementar nuestra propuesta, elaborando un pro grama de trabajo que contribuyera a la recuperación psicosocial de los niños afectados por violencia política.

Entre los años 1994 y 1997 se elaboró, revisó y modificó en varias oportunidades las sesiones y propuestas de trabajo, buscando que respondieran a los objetivos propuestos, que las actividades fueran adecuadas a las edades de los niños (entre 7 y 14 años) y sobretodo que facilitara la expresión de sentimientos y pensamientos.

Una vez comprobada su efectividad con el grupo de niños del Asentamiento Humano Cangalla, en 1997 decidimos validar la propuesta de trabajo con niños con el objetivo de constatar en la práctica si ésta favorecía la recuperación de la salud mental de los niños, saber si podía ser aplicada en diferentes zonas de nuestro país, conocer las condiciones necesarias para aplicar de manera efectiva la pro-puesta y conocer los cambios que se producían en los niños luego de haber pasado por la aplicación de la propuesta. Durante los años 1998 y 1999 se llevó a cabo este proceso y se decidió aplicar la propuesta a una muestra de la población infantil con la que trabajamos, eligiéndose tres zonas de atención del PASMI: Abancay, Ayacucho y Tarapoto ${ }^{1}$.

\section{PUESTA EN MARCHA}

Como primer paso para la validación se elaboró un pequeño módulo de capacitación en la propuesta de trabajo con niños que fue implementado en primer lugar con los miembros de los Equipos Locales y que luego fue adaptado para que éstos pudieran capacitar a sus promotoras, quienes venían trabajando con grupos de niños.

También se elaboraron algunas Fichas de seguimiento que nos darían los elementos necesarios para el análisis, entre ellas elaboramos la Ficha de indicadores de recuperación, que se debía aplicar en tres oportunidades, una antes de iniciar la aplicación del programa, otra durante la aplicación y la última después de haber terminado el trabajo con los niños recogiendo los cambios que se pudieran observar en los niños a lo largo del proceso. La Ficha de datos familiares del niño, que debía ser aplicada al iniciar el trabajo completando alguna información durante el proceso de trabajo con los niños. La ficha de seguimiento a las sesiones que era llenada por un miembro del Equipo Local luego de observar el trabajo de cada promotora y que debía llenarse al terminar cada sesión de trabajo para recoger información sobre cómo funcionaba la sesión, si cumplía los objetivos y si era práctica para el trabajo con los niños. La Ficha de datos de las promotoras y la Ficha de evaluación de metodología de trabajo con niños (para ser aplicada a las promotoras).

\footnotetext{
${ }^{1}$ En la zona de Tarapoto la validación estuvo a cargo del Equipo Local. quienes elaboraron el informe respectivo a partir del trabajo con una población de niños que no consideramos para este trabajo
} 
En Abancay, la aplicación de la propuesta estuvo a cargo de promotoras de las comunidades de Pueblo Joven, Virgen del Rosario, Micaela Bastidas, Intimpas y Ullpuhuayco. Ellas fueron acompañadas por miembros del Equipo Local, quienes se encargaron de orientarlas y conversar cada semana con ellas sobre sus dudas, inquietudes, logros, sugerencias a las sesiones, etc.: en Ayacucho, la propuesta fue aplicada directamente por miembros del Equipo Local en las comunidades de Pampa Hermosa y Señor del Huerto, quienes nos hacían llegar las apreciaciones que tenían en su trabajo directo acerca de la estructura y manejo de las sesiones. Este proceso duró aproximadamente 16 meses y en este tiempo hicimos varias visitas a las zonas para capacitar a los miembros de los equipos locales y para recoger las inquietudes y sugerencias con respecto al manejo y estructura de las sesiones; también se mantuvo estrecha comunicación con los equipos, quienes enviaban por correo la información que recogían en las fichas elaboradas para la validación. Es a partir del análisis de la información recogida que se modifican y/o se corrigen algunas sesiones.

Durante este tiempo se trabajó con una población total de 92 niños cuya edad fluctuaba entre los 7 y 14 años.

A través de la Ficha de datos de los niños pudimos obtener información importante con respecto al grupo de niños. Estos datos fueron los siguientes:

- El 100\% de los niños que participaron en la validación estudiaba en el colegio.

• 90\% de los niños tenían papá y mamá, 9\% tenía sólo mamá y $1 \%$ no tenía padres.

- 58\% vivía en familia nuclear (padre, madre y hermanos) Aquí también se considera a quienes viven con padrastro; $28 \%$ con familia extensa, $8 \%$ con su madre y hermanos, $2 \%$ con su padre y hermanos y $4 \%$ con otros parientes.

- El 60 \% de los niños habían nacido en la ciudad donde se validó la propuesta y el $40 \%$ de ellos habían nacido en otras comunidades.

- 14\% se negó a decir su lugar de procedencia (dónde habían vivido), 86\% manifestó venir de otras provincias o distritos de la región (aproximadamente de 41 lugares diferentes).

- 56\% dijo que el motivo de su salida de la comunidad de origen fue el terrorismo, 25\% dijo que los motivó otras cosas como: conseguir trabajo, educación de los hijos, mejorar la economía y 19\% se negó a decir lo que motivó su salida de la comunidad de origen.

- 14\% no dio razón del tiempo que reside en la zona, 7\% dijo haber vivido en la zona siempre, $10 \%$ vivía en la zona aproximadamente 3 años, 10\% aproximadamente 6 años, $20 \% 9$ años, $15 \%$ vivía en la zona aproximadamente 12 años y $23 \%$ estaba ya 15 años. 
La Ficha de indicadores ${ }^{2}$ fue el instrumento más importante en este proceso, pues al ser aplicada antes, durante y después de la ejecución del programa pudo permitimos observar los cambios que se iban evidenciando en los niños.

En los reportes recogidos por miembros de los Equipos Locales y promotoras/es que aplicaron la propuesta, se señalaban algunas características generales de los niños al iniciar el trabajo; entre ellas: que respondían de manera agresiva frente a una dificultad con sus compañeros, no respetaban los horarios, se llevaban el material a escondidas, no compartían los materiales con sus compañeros, les costaba mucho escucharse mutuamente y contar sus experiencias al promotor/a o al grupo, no conocían muchas cosas acerca de ellos mismos (fecha de nacimiento, apellidos completos, etc) y de su familia como por ejemplo nombres de sus abuelos, el nombre de su comunidad de origen, vivencias de sus familias. entre otras cosas. Hubieron también algunos niños que mostraban características opuestas, eran demasiado callados no pedían ayuda ni preguntaban, se dejaban quitar las cosas, nunca se quejaban de nada y les costaba mucho integrarse al grupo.

\section{RESULTADOS}

Con respecto a los cambios observados a través de la Ficha de indicadores, pudimos encontrar que el área en la que mostraron mejoría fue la de Identidad, donde un $71 \%$ de los niños lograron mostrar cambios favorables, especialmente con respecto al reconocimiento de su comunidad de origen, donde $99 \%$ de los niños respondieron positivamente. También se pudo observar que el $63 \%$ de los niños, rápidamente mostraron cambios relacionados a la Expresión de pensamientos y sentimientos, logrando contar al grupo algunas vivencias y cómo se sintieron con relación a ellas (esto se logró en $83 \%$ de los niños), así como lo que pensaban y sentían con respecto a ellos mismos (84\%).

En la Relación con su comunidad los cambios que el 95\% de los niños evidenciaron rápidamente fueron con relación al interés por cuidar las cosas de su comunidad; un 83\% mostró también mayor interés por conocer más sobre su comunidad y lo que pasaba en ella (labores comunales, asambleas, decisiones, etc.), mientras que el $81 \%$ mostró interés en participar de algunas actividades o celebraciones comunales

El área en la que los niños tuvieron más dificultades fue la de valoración personal, pudiéndose observar que el proceso fue mucho mas lento con relación al cuidado de su apariencia personal donde un $68 \%$ de los niños mostró cambios favorables y 87\% incremento su preocupación por el cuidado de sus pertenencias.

Otras áreas en las que el proceso de cambio fue lento estaban referidas a Relaciones interpersonales donde el 56\% de los niños lograron cambios positivos en los indicadores y el 60\% lograron cambios en el área visión de futuro. En la primera el aspecto que más difícil resultó para los niños, fue el de controlarse y no agredir cuando los fastidiaban y en la segunda el poder plantearse metas para un futuro cercano.

Es importante señalar que estos resultados fueron conseguidos gracias también a las actitudes positivas de las personas que dirigían los grupos y al buen manejo de las sesiones

\footnotetext{
${ }^{2}$ El modelo de ficha de indicadores aplicada en este proceso de validación se encuentra en el anexo del documento
} 
que gracias al acompañamiento de los miembros de los equipos locales fue mejorando a lo largo de la aplicación del programa.

A continuación los cuadros y gráficos estadísticos que ilustran los resultados expuestos.

Tabla $\mathbf{N}^{\circ} 1$

Resultados de la primera evaluación de indicadores en función al número de niños $(N=92)$

\begin{tabular}{|c|c|c|c|c|c|c|c|c|c|c|c|c|c|c|c|c|c|c|}
\hline & \multicolumn{6}{|c|}{ INICIO } & \multicolumn{6}{|c|}{ PROCESO } & \multicolumn{6}{|c|}{ LOGRO } \\
\hline$\frac{\mathrm{A}}{\mathrm{l}}$ & 1 & 11 & 1111 & TV & V & $\nabla I$ & 1 & $\Pi 11$ & III & TV & V & VI & $T$ & II & III & IV & $\nabla$ & VI \\
\hline $\mathrm{a}$ & 26 & 32 & 15 & 55 & 52 & 23 & 26 & 38 & 27 & 2 & 19 & 37 & 40 & 22 & 50 & 35 & 21 & 32 \\
\hline $\mathrm{b}$ & 32 & 58 & 33 & 34 & 63 & 57 & 42 & 21 & 32 & 10 & 14 & 15 & 18 & 13 & 27 & 58 & 15 & 20 \\
\hline $\mathrm{c}$ & 34 & 71 & 11 & 54 & 80 & 64 & 33 & 11 & 44 & 1 & 6 & 10 & 25 & 10 & 37 & 37 & 6 & 18 \\
\hline $\mathrm{d}$ & 26 & 54 & 29 & 74 & & 49 & 38 & 29 & 29 & 0 & & 28 & 28 & 9 & 34 & 18 & & 15 \\
\hline $\mathrm{e}$ & 34 & 41 & 13 & & & & 27 & 28 & 39 & & & & 31 & 23 & 40 & & & \\
\hline $\mathrm{f}$ & 49 & 18 & 32 & & & & 27 & 39 & 21 & & & & 16 & 35 & 30 & & & \\
\hline $\mathrm{g}$ & & & 19 & & & & & & 21 & & & & & & 52 & & & \\
\hline
\end{tabular}

Tabla $N^{\circ}$

Resultados de la evaluación final de indicadores en función al número de niños $(N=92)$

\begin{tabular}{|c|c|c|c|c|c|c|c|c|c|c|c|c|c|c|c|c|c|c|}
\cline { 2 - 18 } \multicolumn{1}{c|}{} & \multicolumn{9}{c|}{ INICIO } & \multicolumn{6}{c|}{ PROCESO } & \multicolumn{6}{c|}{ LOGRO } \\
\hline A & I & II & III & IV & V & VI & I & II & III & IV & V & VI & I & II & III & IV & V & VI \\
\hline a & 0 & 1 & 0 & 0 & 52 & 0 & 2 & 16 & 7 & 4 & 8 & 5 & 90 & 75 & 85 & 88 & 83 & 87 \\
\hline b & 0 & 1 & 0 & 0 & 63 & 0 & 16 & 15 & 10 & 1 & 15 & 15 & 76 & 76 & 82 & 91 & 76 & 77 \\
\hline c & 0 & 1 & 0 & 1 & 80 & 0 & 7 & 14 & 4 & 1 & 23 & 17 & 85 & 77 & 88 & 90 & 66 & 75 \\
\hline d & 0 & & 0 & 0 & & 2 & 2 & 14 & 9 & 23 & & 16 & 90 & 77 & 83 & 69 & & 74 \\
\hline e & 0 & 0 & 0 & & & & 12 & 21 & 2 & & & & 80 & 71 & 90 & & & \\
\hline f & 0 & 0 & 0 & & & & 30 & 6 & 29 & & & & 61 & 86 & 63 & & & \\
\hline g & & & 0 & & & & & & 12 & & & & & & 80 & & & \\
\hline
\end{tabular}




\section{Grafico $\mathrm{N}^{\circ} 1$}

Gráficos por áreas, comparando resultados de la primera y última evaluación por ítem de

AREA I : RELACIONES INTERPERSONALES

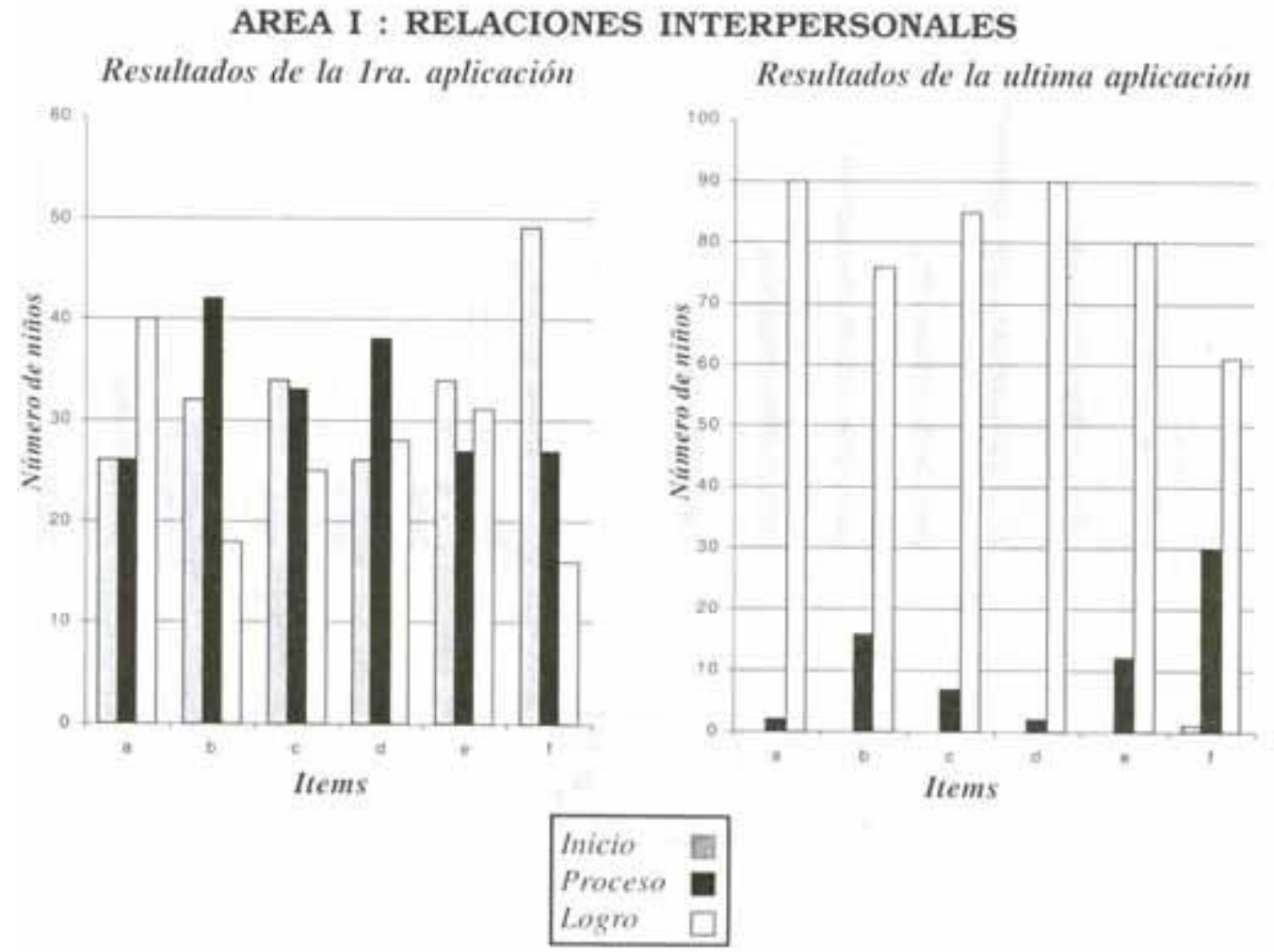

cada área

\section{AREA II : EXPRESION DE PENSAMIENTOS Y SENTIMIENTOS}

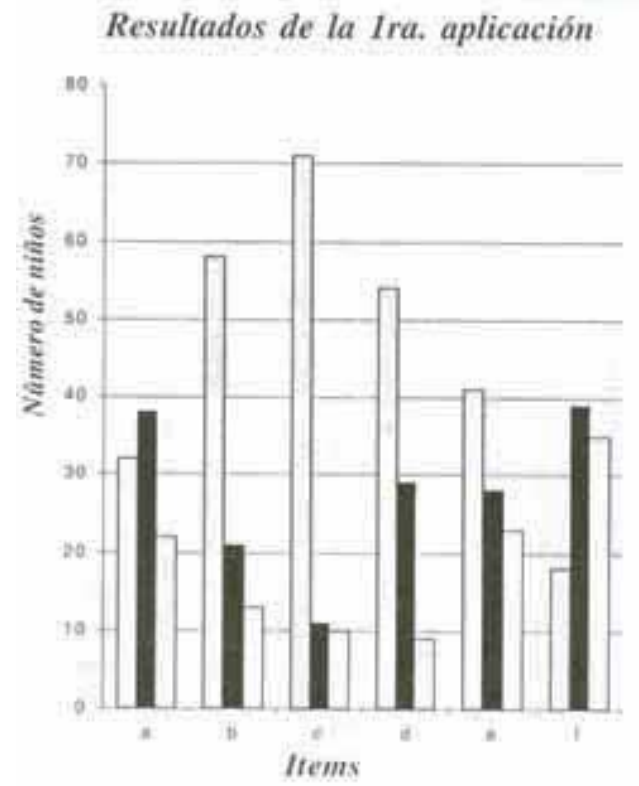

Resultados de la ultima aplicación

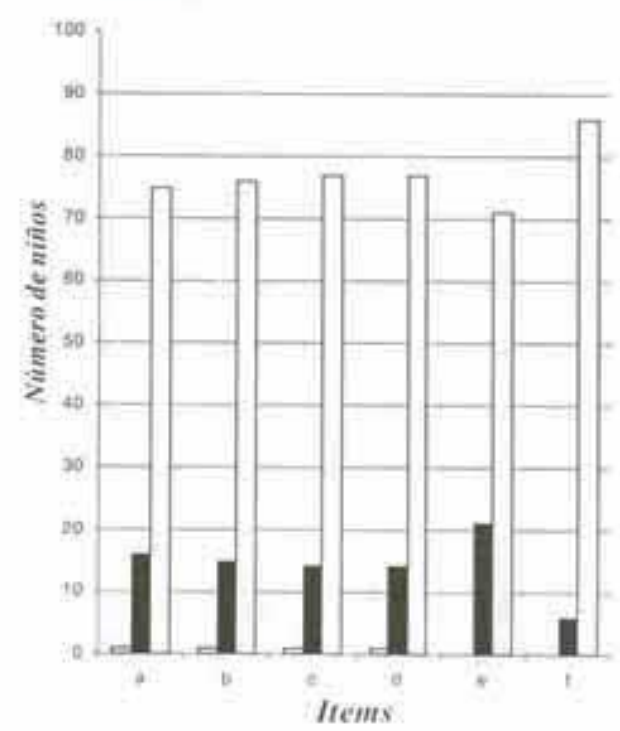


AREA III : VALORACION PERSONAL


\begin{tabular}{|l|}
\hline Inicio \\
Proceso \\
Logro \\
\hline
\end{tabular}

AREA IV : IDENTIDAD

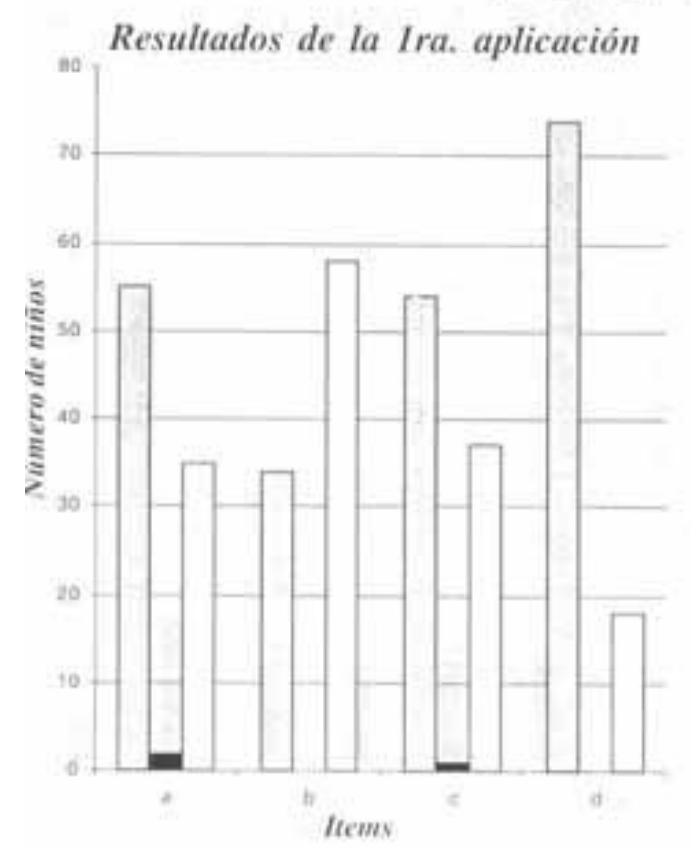

Resultados de la ultima aplicación

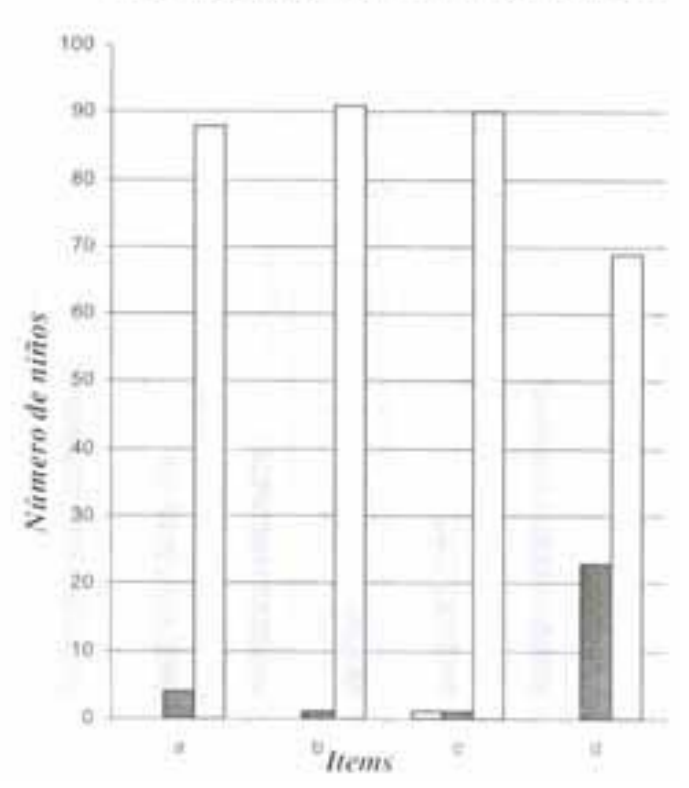


AREA V : VISION DE FUTURO

Resultados de la Ira. aplicaciôn

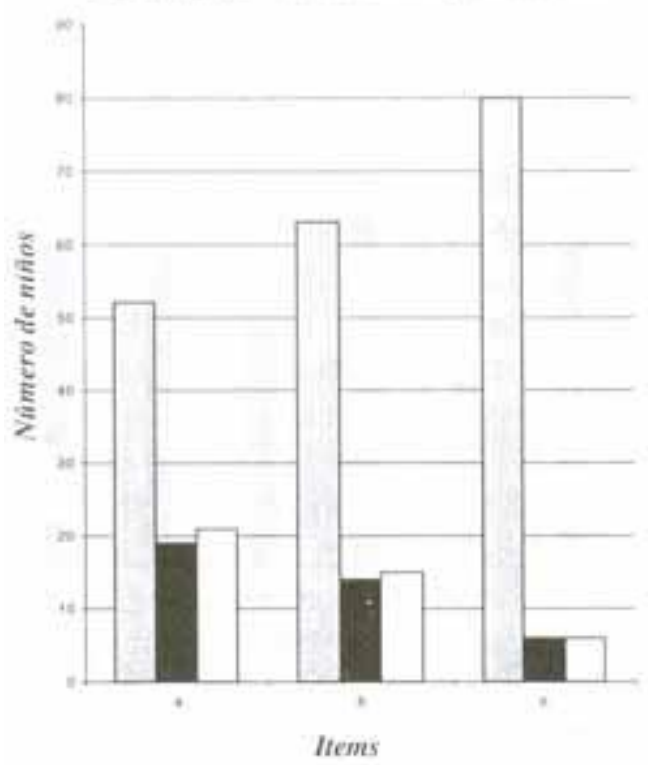

Resultados de la ultima aplicación



Inicio 팀

Proceso

Logro

\section{AREA V : VISION DE FUTURO}

Resultados de la Ira. aplicación

Resultados de la ultima aplicación
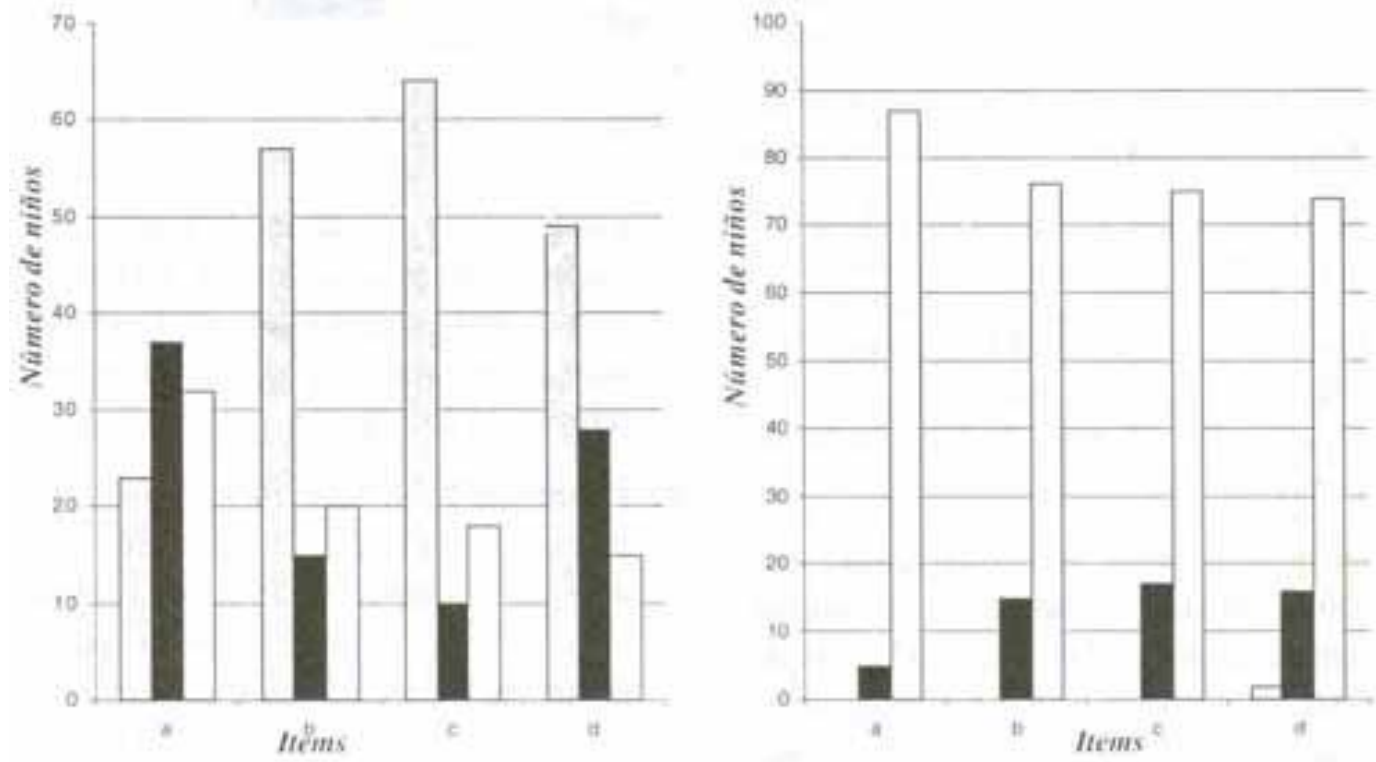
Tabla No. 3

Resultados de niveles de recuperación por areas en función del númeo de niños

\begin{tabular}{|c|c|c|c|c|}
\hline Area & Inicio & Proceso & Logro & Total \\
\hline I & 4 & 37 & 51 & 92 \\
II & 14 & 20 & 58 & 92 \\
III & 4 & 39 & 49 & 92 \\
IV & 8 & 27 & 65 & 92 \\
V & 3 & 34 & 55 & 92 \\
VI & 8 & 35 & 57 & 92 \\
\hline
\end{tabular}

Inicio

Proceso

Logro

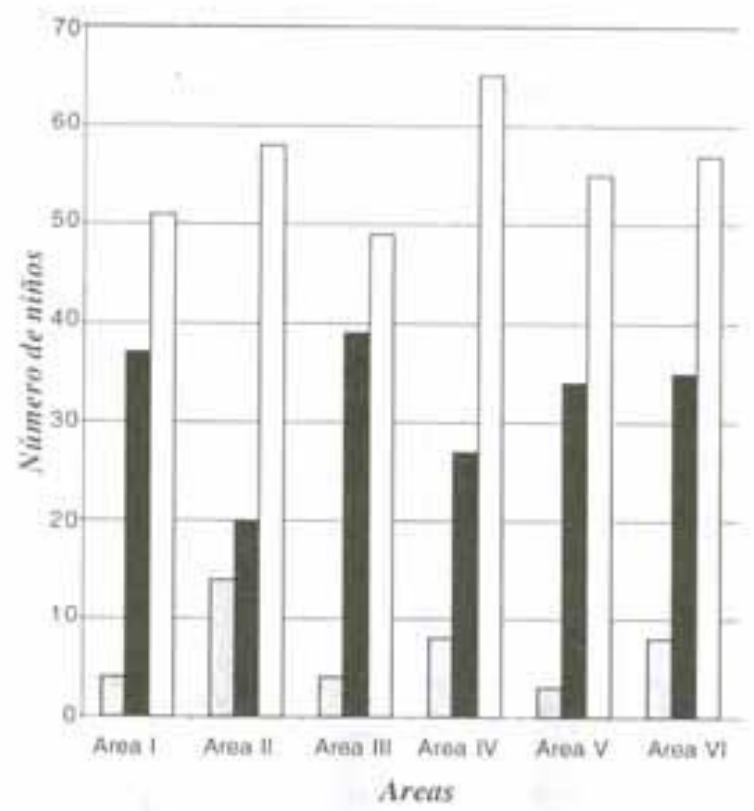

Tabla No. 4

Resultados de niveles de recuperación por categorías

\begin{tabular}{|l|c|c|c|}
\hline Categorias & Inicio & Proceso & Logro \\
\hline Niños & 1 & 36 & 55 \\
Porcentaje & $1 \%$ & $39 \%$ & $60 \%$ \\
\hline
\end{tabular}

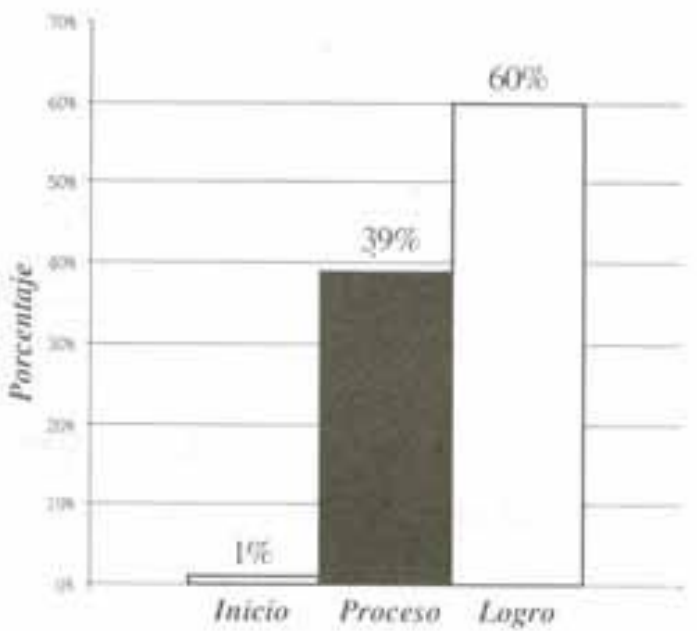

\section{CONCLUSIONES}

1. Se mostraron cambios a nivel de todas las áreas evaluadas.

2. Las áreas en las que los cambios fueron más evidentes fueron el Área IV Identidad y el Área II Expresión de Pensamientos y Sentimientos

3. El área de Identidad fue en la que más niños mostraron cambios al parecer debido a que en las actividades de referidas a este tema los niños se entusiasmaban más por conversar con sus padres (cosa que antes no hacían o lo hacían muy poco), por conocer cosas acerca de la comunidad de donde venían y por saber qué vivencias tuvo su familia en esa época.

4. El área de Expresión de pensamientos y sentimientos fue la que empezó a mostrar cambios más rápidamente. Creemos que esto se debió a que se puso mucho énfasis en que la primera etapa de trabajo la promotora ganara la confianza del grupo, además las actitudes de respeto, escucha, cercanía entre otras aunadas a las técnicas de arte facilitaron que los niños sientan que el espacio les permitía expresarse.

5. El área de valoración personal fue en la que menos logros se evidenciaron debido a que 
esta es una de las áreas más afectadas en los niños que han pasado situaciones de violencia, además hay una fuerte influencia del hogar y de la escuela, espacios que si no son sensibilizados para modificar su tipo de relación con los niños (menos castigadoras y represivas) serán siempre un obstáculo para el trabajo. En suma a partir de los resultados encontrados se consideró de suma importancia incidir con más fuerza en el trabajo con familia y escuela, así como en el trabajo con comunidad y dar mayor fuerza al trabajo en el área de valoración personal.

6. Hubo un alto número de niños que según los indicadores quedaban en proceso de logro de los cambios esperados, por lo que se continuó el trabajo a partir de sesiones complementarias que respondían a los mismos objetivos con actividades diferentes y que estaban dirigidas a reforzar las áreas débiles.

7. Se constató que un elemento importante a desarrollar en el trabajo con niños es la formación adecuada de las personas que se hagan cargo de los grupos. Esta formación tiene que ver con dos niveles: La capacitación (de acuerdo a la problemática que se atienda), que incorpore también la metodología de trabajo con niños y la formación humana que tome en cuenta el que los adultos procesen sus conflictos y vivencias y fortalezcan sus recursos para ser soporte y referentes positivos para los niños. Esto tiene que ver mucho con el cambio de actitudes.

8. En conclusión, podemos decir por testimonios de miembros de los Equipos Locales y promotoras y por los resultados finales de la validación que al finalizar la aplicación del programa los niños mostraron cambios los cuales se fueron evidenciando gradualmente a lo largo de la aplicación de la propuesta. Aprendieron a respetar el horario y las normas de convivencia establecidas por ellos mismos, aprendieron a compartir los materiales, cuidar las cosas del taller, integrarse como grupo; aprendieron a respetar el momento para compartir lo trabajado y sus vivencias, llegaron a conocerse mejor identificando sus características positivas y negativas, tuvieron oportunidad de conocer mejor a sus padres, la historia de su familia y su comunidad de origen.

\section{BIBLIOGRAFIA}

CEDAPP. Centro de Desarrollo y Asesoría Psicosocial. «Infancia y violencia 2». Ediciones CEDAPP. Serie Reflexiones en Psicología 5. Lima, 1994

Silva, Giselle. «Resiliencia y Violencia Política en Niños».Universidad Nacional de Lanus. Buenos Aires, 1999.

Villapolo, Leslie; Vásquez, Norma. «Entre el juego y la guerra». Ediciones CAAAP Centro Amazónico de Antropología y Aplicación Práctica. Lima, 1999.

Avensur Félix, Lili; Bustamante Soto, Elsa; Nieto Degregori, Rocío y Padilla Marroquín, Daphne. «Elementos básico para nuestra formación». Serie Salud Mental y Violencia Política. REDINFA Red para la Infancia y la Familia - Perú. Lima, 2000 


\section{FICHA DE INDICADORES}

Nombre de la promotora:

Nombre del niño:

Dónde esta ubicado el taller del niño:. fecha:.......



\section{$\mathrm{S}=\mathrm{Si} \quad \mathrm{A}=\mathrm{A}$ veces $\quad \mathrm{N}=\mathrm{No}$}

Es importante que esta ficha sea aplicada tres veces durante el tiempo que se trabaje con los años. La primera aplicación debe hacerse antes de iniciar el trabajo con el grupo, cuando aun no se ha tenido actividades de ningún tipo con ellos.

La segunda aplicación puede hacerse a mitad del programa, y la tercera aplicación cuando ya hayan finalizado. 